\title{
Hyperdiploidy defines a distinct cytogenetic entity of meningiomas
}

\author{
Ralf Ketter • Yoo-Jin Kim - Simone Storck • \\ Jörg Rahnenführer · Bernd F. M. Romeike • \\ Wolf-Ingo Steudel · Klaus D. Zang · Wolfram Henn
}

Received: 22 November 2006/ Accepted: 11 December 2006/Published online: 17 January 2007

(C) Springer Science+Business Media B.V. 2007

\begin{abstract}
Background The most common chromosomal aberration found in meningiomas is monosomy 22. Progression and recurrence of meningiomas are usually associated with additional chromosome losses. Rarely, however, meningiomas have strongly hyperdiploid karyotypes with over 50 chromosomes; the objective of this study was to explore the cytogenetic and histopathologic patterns as well as the clinical significance of hyperdiploidy in meningiomas.

Methods Within a series of 677 consecutive meningiomas, we identified a subgroup comprising 16 cases that display a strikingly uniform pattern of hyperdiploidy mostly without structural chromosome rearrangements, as shown by banding techniques
\end{abstract}

R. Ketter and Y.-J. Kim have contributed equally to this work.

R. Ketter · W.-I. Steudel

Neurosurgical Clinic, Saarland University,

Homburg/Saar, Germany

Y.-J. Kim · B. F. M. Romeike

Institute of Neuropathology, Saarland University,

Homburg/Saar, Germany

S. Storck - K. D. Zang · W. Henn

Institute of Human Genetics, Saarland University,

Homburg/Saar, Germany

J. Rahnenführer

Max Planck Institute for Informatics,

66123 Saarbruecken, Germany

R. Ketter $(\square)$

Department of Neurosurgery, Saarland University,

66421 Homburg/Saar, Germany

e-mail: ncrket@uniklinik-saarland.de and, in the single structurally aberrant case, spectral karyotyping.

Results These meningiomas each have between 50 and 56 chromosomes, with trisomy 12 (14/16 cases), trisomy 20 (13/16 cases), trisomy 5 (12/16 cases), and trisomy 17 (10/16 cases). Histomorphologically, hyperdiploid meningiomas feature a heterogeneous phenotype. However, they are associated with a higher histological grade, and decreased expression of alkaline phosphatase as compared to meningiomas with typical karyotype. In two patients, recurrences were documented and three patients died of disease during the period of observation, indicating a worse prognosis of hyperdiploid than of cytogenetically typical meningiomas.

Conclusion We conclude that hyperdiploidy constitutes a small but clinically relevant entity of biologically aggressive meningiomas, which are cytogenetically distinguishable from the majority of commontype meningiomas.

Keywords Meningioma - Cytogenetics . Hyperdiploidy · Progression

\section{Introduction}

Meningiomas, which arise from the arachnoidal layer of the brain coverings, account for about $20 \%$ of primary tumors of the central nervous system. Although meningiomas are usually benign WHO grade I tumors, about $10 \%$ of meningiomas are more aggressive (grades II or III) and tend to recur [1]. The most common primary cytogenetic feature of all grades of meningioma is monosomy 22, whereas tumor 
progression and recurrence is associated with stronger hypodiploidy, in particular monosomy of chromosomes $14 \mathrm{and} /$ or 18, and loss of the short arm of a chromosome 1 [2-6]. Hyperdiploidy, on the other hand, has long been known to be found in rare instances of meningiomas $[2,7,8]$. Beyond these anecdotal reports of karyotypes, hyperdiploidy in meningiomas has been demonstrated so far mainly through FISH with probes for single chromosomes, such as \# 6, 7, 10, 17, 20, 22, and X [9-11]. While gains of chromosome 22 have been shown to be associated with aggressive clinical features of meningiomas [12,13], no conclusive data is at hand so far on the overall pattern of chromosome gains in hyperdiploid meningiomas.

We here present a series of 16 markedly hyperdiploid meningiomas, taken from a long-term consecutive study on 677 cytogenetically characterized meningiomas, which display a non-random pattern of trisomies and tetrasomies, along with absence of the otherwise typical monosomy 22 in all but two cases. The hyperdiploid karyotype of these tumors is shown to be associated with a more aggressive behavior, thus defining hyperdiploid meningiomas as a clinically important cytogenetic tumor entity.

\section{Materials and methods}

\section{Patients}

This retrospective study was composed of 16 patients [13 women (w) and 3 men $(\mathrm{m})$ ] with hyperdiploid meningiomas who underwent surgery at the Department of Neurosurgery, Saarland University, between January 1973 and April 2005. The mean age \pm SD was $58.8 \pm 13.2$ years. The mean age of the female patients was $57.4 \pm 14.1$ years and that of the male patients was $65 \pm 5.6$ years. Written informed consent was obtained from each patient participating in the study.

The group of these 16 hyperdiploid meningiomas was compared with a control group of meningiomas ( $n=661$ ) belonging to the following genetic progression score (GPS-see Sect. "Statistical analysis") groups:

- meningiomas with normal chromosome set (GPS group $0 ; n=280$ ),

- meningiomas with monosomy 22 as the sole cytogenetic aberration (GPS group 1; $n=241$ ),

- markedly hypodiploid meningiomas with or without $1 \mathrm{p}$-, i.e., deletions of the short arm of a chromosome 1 (GPS group 2; $n=140$ ).
The mean age in the comparison group was 57.3 years $(\mathrm{SD} \pm 12.8$ years $)$.

\section{Clinical variables}

The clinical variables included patient sex and age, tumor location, and the completeness of tumor extirpation.

According to Olivecrona [14], we defined the following eight groups with respect to the location of the meningioma: convexity, parasagittal region, tuberculum sellae, olfactory groove, sphenoid wing ridge, posterior cranial fossa, tentorium, and spinal canal. The classification was made on the basis of a review of the preoperative CT and MR images.

Complete surgical extirpation of the tumor was defined as Simpson grade I or II, corresponding to a macroscopically determined complete tumor resection with bipolar coagulation of the dural insertion [15].

Postoperatively, the patients were examined in our neurosurgical outpatient department either at scheduled follow-up intervals or when neurological symptoms had appeared. Two patients with hyperdiploid meningiomas were lost to follow-up after 1 month, one of these died within 1 month after resection. The mean period of observation of patients with hyperdiploid meningiomas was 60.1 months and 40.3 months for the control group, respectively.

\section{Cytogenetic and histological analysis}

Tissue specimens from tumors were obtained freshly after surgery and processed according to previously described methods [16]. The cell cultures were grown in Dulbecco's modified Eagle's minimal essential medium supplemented with $10 \%$ fetal calf serum and antibiotics. Chromosome preparation from primary cultures, GTG-banding, and karyotyping followed standard procedures. Spectral karyotyping (SKY) of metaphase spreads was performed using an ASI SpectraCube (Applied Spectral Imaging) according to standard protocols [17].

Formalin fixed and paraffin embedded sections of 15 meningiomas were used for histomorphological analysis and immunohistochemical staining for Ki-67 (monoclonal antibody Ki-S5, DAKO Cytomation, Denmark; 1:50, microwave). For case no. 2, no material for histopathologic examination was available. The following histological parameters indicating a greater likelihood of recurrence and/or aggressive behavior [18-20] were evaluated for each of the 15 tumors: loss of lobular structure, increased mitotic 
activity, prominent nucleoli, increased cellularity, small cells with high nuclear/cytoplasmic ratio, foci of spontaneous necrosis, and brain invasion. In nine cases of the hyperdiploid group, histochemical detection of alkaline phosphatase was performed in cryostat sections with hexazotized triamino-tritolyl-methane-chloride (Neufuchsin; Merck, Germany) as described previously [21]. The Ki-67 labeling index (LI)—defined as the percentage of immunostained nuclei divided by the total number of nuclei-was assessed for each of those 15 hyperdiploid meningiomas by means of an image analysis program based on ImageJ developed by one of the authors [22]. Tumor grading was determined for all hyperdiploid and control meningiomas according to the criteria defined by the World Health Organization [18].

\section{Statistical analysis}

\section{Oncogenetic tree models}

Mixtures of oncogenetic trees were used to describe the ordered accumulation of genetic aberrations during tumor progression. In a single oncogenetic tree, vertices represent genetic events and edges between vertices represent transitions between the events. Each edge is associated with the probability that the successor event will occur, given the predecessor event has already occurred. In the model genetic events are assumed to be non-reversible, thus the disease process can be fully described by the accumulation of genetic aberrations. Every tumor is assigned to a specific tree according to the relative likelihood that its genetic pattern was generated by this tree. The mixture weights associated with the single trees then represent the sum over all tumors. The weight of the star-like tree thus indicates the proportion of tumors whose progression is not described by the other estimated components. Oncogenetic trees mixture models can be estimated from data consisting of genetic patterns with an Expectation-Maximization algorithm introduced by Beerenwinkel et al. [23, 24].

\section{Genetic progression score}

In oncogenetic tree models consecutive genetic aberrations are associated with corresponding conditional transition probabilities. These probabilities can be converted to average waiting times, by assuming Poisson processes for the occurrence of aberrations, see Rahnenführer et al. [25] for details. The GPS of a tumor then is defined as the average waiting time of its pattern of genetic aberrations, given the underlying tree mixture model. Tumors with few aberrations that appear early in the tree model receive low GPS values, whereas tumors with many late aberrations in the tree model are associated with higher GPS values.

\section{Results}

\section{Clinical findings}

Among patients with hyperdiploid meningiomas, female patients predominated over male patients, with a sex ratio of 4.33:1. On average, male patients were slightly but not significantly older than female patients. Table 1 gives an overview of the clinical data of the meningiomas and the karyotypes established from the tumor samples.

Thirteen of the 16 hyperdiploid tumors were located at the convexity and parasagittal region. Only three were located at the skull base, and no tumor at the spine. The preference of the convexity in the group of hyperdiploid meningiomas was not significantly higher than in the cytogenetically typical meningiomas $(p=0.54)$.

We found two cases of recurrence after macroscopically total tumor removal according to Simpson grade I. The two recurrent cases in this study represent one male and one female patient. One case (no. 15) recurred after 14 months, and the other (no. 10) after 74 months of follow-up. In case no. 9 after surgical extirpation of the tumor, a radiation with $60 \mathrm{~Gy}$ to the former tumor region was applied and no recurrence arose after 16 years of follow-up. In our control group recurrence was observed only in $6.2 \%$ of meningiomas of GPS groups 0 and 1 but in $33.3 \%$ of tumors belonging to the GPS group $2\left(p=2.1 \times 10^{-8}\right)$. In the present investigation of the hyperdiploid meningiomas $12.5 \%$ of the cases showed recurrence. Compared to our control group there was a tendency toward an elevated recurrence rate but the difference was not significant $(p=0.94)$.

Table 2 gives an overview of histomorphological features of hyperdiploid meningiomas. The outstanding features in all hyperdiploid meningiomas, except cases no. 12 and 15, were patternless growth with loss of lobular pattern or microcystic degeneration. In detail, five tumors presented a predominantly microcystic differentiation, eight tumors were classified as "syncytial" subtype with patternless growth, including six atypical meningiomas out of nine atypical meningiomas in the overall cohort. In two cases (no. 8 and 14) a persistent epitheloid appearance of tumor cells with 
Table 1 Clinical and cytogenetic data of 16 hyperdiploid meningiomas

\begin{tabular}{|c|c|c|c|c|c|c|}
\hline $\begin{array}{l}\text { Case } \\
\text { no. }\end{array}$ & $\begin{array}{l}\text { Sex/ } \\
\text { age }\end{array}$ & Site & $\begin{array}{l}\text { Simpson } \\
\text { grade }\end{array}$ & $\begin{array}{l}\text { Follow-up } \\
\text { months }\end{array}$ & $\begin{array}{l}\text { Course } \\
\text { of } \\
\text { disease }\end{array}$ & Karyotype \\
\hline 1 & $\mathrm{~m} / 72$ & 4 & II & 58 & $\mathrm{~d}$ & $\begin{array}{l}54, \mathrm{X},-\mathrm{Y}, \mathrm{t}(4 ; 5)(\mathrm{q} 35 ; \mathrm{q} 12),+5,+7,+9,+11,+12,+13 \\
\quad+17,+18,+20[\mathrm{cp} 14]\end{array}$ \\
\hline 2 & $\mathrm{f} / 47$ & 1 & I & 191 & - & $56, \mathrm{X},-\mathrm{X},+4,+5,+8,+12,+14,+15,+17,+18,+19,+20[\mathrm{cp} 18]$ \\
\hline 3 & $\mathrm{f} / 70$ & 4 & II & 35 & - & $54, \mathrm{XX},+3,+4,+5,+9,+12,+13,+15,+20[\mathrm{cp} 8]$ \\
\hline 4 & $\mathrm{f} / 69$ & 1 & I & 32 & $\mathrm{~d}$ & $51, \mathrm{XX},+3,+5,+12,+13,+\operatorname{mar}[\mathrm{cp} 11]$ \\
\hline 5 & $\mathrm{~m} / 60$ & 1 & I & 14 & - & $\begin{array}{l}56, \mathrm{XY},+3,+4,-7,+10,+12,-13,+16,+19,+20,+20, \\
\quad+4 \operatorname{mar}[\operatorname{cp} 16]\end{array}$ \\
\hline 6 & $\mathrm{f} / 65$ & 1 & I & 1 & $\mathrm{~d}$ & $\begin{array}{l}62, \mathrm{XX},+5,+5,+6,+7,+8,+9,+11,+13,+13,+16 \\
\quad+17,+18,+18,+19,+20,+22[2]\end{array}$ \\
\hline 7 & $\mathrm{f} / 67$ & 1 & III & 1 & SD & $51, \mathrm{XX},+12,+13,+16,+17,+20[8]$ \\
\hline 8 & $\mathrm{f} / 65$ & 1 & I & 36 & - & $54, \mathrm{XX},+3,+8,+9,+12,+13,+15,+20,+20[11]$ \\
\hline 9 & $\mathrm{f} / 42$ & 2 & III & 170 & SD & $50, \mathrm{XX},+4,+12,+17,+20[\mathrm{cp} 17]$ \\
\hline 10 & $\mathrm{f} / 61$ & 1 & I & 14 & $\mathrm{r}$ & $52, \mathrm{XX},+5,+10,+12,+15,+17,+20[16]$ \\
\hline 11 & $\mathrm{f} / 56$ & 1 & I & 35 & - & $\begin{array}{l}59, \mathrm{XX},+4,+5,+5,+6,+7,+11,+12,+13,+16 \\
\quad+17,+18,+19,+20[3]\end{array}$ \\
\hline 12 & $\mathrm{f} / 23$ & 2 & I & 42 & - & $52, \mathrm{XX},+3,+5,+9,+10,+12,+17,-22[12]$ \\
\hline 13 & $\mathrm{f} / 73$ & 1 & I & 24 & - & $53, \mathrm{XX},+3,+5,+8,+9,+12,+13,+20[\mathrm{cp} 5]$ \\
\hline 14 & $\mathrm{f} / 54$ & 1 & I & 24 & - & $53, \mathrm{XX},+5,+7,+10,+10,+12,+16,+17[15]$ \\
\hline 15 & $\mathrm{~m} / 64$ & 2 & I & 89 & $\mathrm{r}$ & $50, \mathrm{XY},+3,+5,+12,+17,+20,-22[\mathrm{cp} 16]$ \\
\hline 16 & $\mathrm{f} / 72$ & 3 & II & 46 & - & $\begin{array}{l}56, \mathrm{XXX},+3,+4,+5,+8,+9,+12,-13,-16 \\
\quad+\operatorname{dic}(13 ; 16)(\mathrm{p} 13 ; \mathrm{p} 13),+17,+20,+20[\mathrm{cp} 10]\end{array}$ \\
\hline
\end{tabular}

Site -1 convexity, 2 parasagittal region, 3 olfactory groove, 4 sphenoid wing ridge; course of disease- $r$ recurrence, $d$ dead of disease, $S D$ stable disease

sharply marked cell borders was observed. Occasional epitheloid patterns were also seen in cases no. 4,7 , and 11 (Fig. 1a). The microcystic meningiomas in particular had numerous blood vessels. The vascular channels were either thin-walled or had hyalinized, thickened walls. All of the three meningiomas at the skull base showed patternless growth without or only slight microcystic degeneration. The cases no. 12 and 15 featured a persistent storiform growth pattern and were classified as fibrous meningiomas (Fig. 1b). In the cases no. 7 and 14, brain invasion was verifiable. Necrosis in the absence of prior embolization were

Table 2 Histopathological features in 15 hyperdiploid meningiomas (except case no. 2, which was not available for evaluation)

\begin{tabular}{|c|c|c|c|c|c|c|c|c|c|c|c|c|}
\hline $\begin{array}{l}\text { Case } \\
\text { no. }\end{array}$ & $\begin{array}{l}\text { WHO } \\
\text { grade }\end{array}$ & $\begin{array}{l}\text { Patternless } \\
\text { growth }\end{array}$ & Microcystic & $\begin{array}{l}\text { Fibrous } \\
\text { pattern }\end{array}$ & $\begin{array}{l}\text { Prominent } \\
\text { nucleoli }\end{array}$ & $\begin{array}{l}\text { Mitotic } \\
\text { index }^{\mathrm{a}}\end{array}$ & Necrosis & $\begin{array}{l}\text { High } \\
\text { nuclear: } \\
\text { cytoplasm } \\
\text { ratio }\end{array}$ & $\begin{array}{l}\text { Hyper- } \\
\text { cellularity }\end{array}$ & $\begin{array}{l}\text { Brain } \\
\text { invasion }\end{array}$ & $\begin{array}{l}\text { AP } \\
\text { activity }\end{array}$ & $\begin{array}{l}\text { Ki-67 } \\
\text { LI (\%) }\end{array}$ \\
\hline 1 & I & + & ++ & - & - & 0 & - & - & + & - & n.a. & 3.5 \\
\hline 3 & II & ++ & - & + & + & 1 & - & - & + & - & - & 3.4 \\
\hline 4 & II & ++ & + & - & - & 1 & - & ++ & ++ & - & n.a. & 4.3 \\
\hline 5 & II & + & ++ & + & ++ & 4 & - & - & - & - & n.a. & 4.5 \\
\hline 6 & I & - & ++ & + & - & 0 & - & - & - & - & n.a. & 3 \\
\hline 7 & II & ++ & - & - & + & 2 & + & ++ & ++ & ++ & $+/-$ & 13.5 \\
\hline 8 & I & ++ & + & - & - & 1 & - & - & - & - & $+/-$ & 4.1 \\
\hline 9 & I & + & ++ & - & - & 0 & - & - & - & - & n.a. & 3.2 \\
\hline 10 & I & ++ & + & - & - & 1 & - & - & - & - & n.a. & 1.1 \\
\hline 11 & I & + & ++ & - & ++ & 1 & - & - & - & - & $+/-$ & 11.3 \\
\hline 12 & II & - & - & ++ & + & 2 & ++ & ++ & + & - & - & 11.9 \\
\hline 13 & II & ++ & + & - & + & 4 & - & - & + & - & - & 4.5 \\
\hline 14 & II & ++ & + & - & ++ & 1 & - & + & ++ & ++ & $+/-$ & 8.2 \\
\hline 15 & II & - & - & ++ & ++ & 0 & - & + & + & - & $+/-$ & 6.0 \\
\hline 16 & II & ++ & - & - & - & 2 & + & + & - & - & $+/-$ & 3.3 \\
\hline
\end{tabular}

"_" represents absent, "+" represents present, "++" represents predominant, "+/-" represents focal loss of activity of alkaline phosphatase

n.a. not assessed

${ }^{\text {a }}$ Mitoses/10 high power fields 
Fig. 1 (a) Typical histomorphology of a hyperdiploid meningioma with syncytial areas, microcystic degeneration, and epitheloid appearance; (b) storiform growth pattern (case no. 12); (c) partial loss of alkaline phosphatase activity; (d) moderately increased Ki-67 LI (case no. 14)

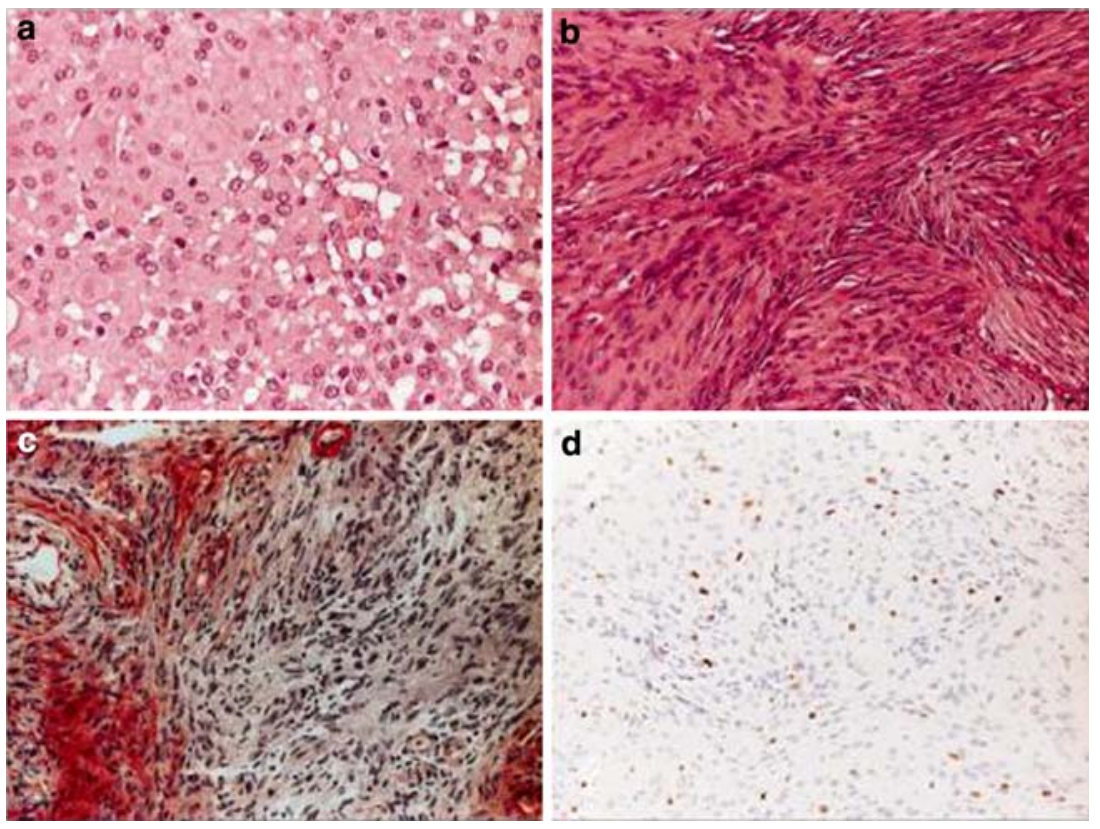

found in three meningiomas (cases no. 7, 12, and 16). Distribution of histopathological parameters, indicating a more aggressive biological behavior for each of the cytogenetic subgroups are displayed in Table 3. The likelihood for higher grade of malignancy, and loss of expression of alkaline phosphatase were significantly elevated for hyperdiploid meningiomas compared to meningiomas with typical karyotype and were similar to the findings in meningiomas with markedly hypodiploid karyotypes without loss of $1 \mathrm{p}$. The overall mean Ki-67 LI \pm SD of the investigated hyperdiploid meningiomas was $5.7 \% \pm 3.6$ and ranged from $1.1 \%$ (case no. 10) to $13.5 \%$ in case no. 7 (Table 2).

Cytogenetically, the hyperdiploid meningiomas comprise about $2.4 \%$ of all karyotyped meningiomas within our collection. They are characterized by trisomies, rarely tetrasomies, of various autosomes, almost uniformly in the absence of structural aberrations (Fig. 2a, b). An apparent structural chromosome rearrangement was encountered only in case no. 16;
SKY analysis confirms a $\operatorname{dic}(13 ; 16)(\mathrm{p} 13 ; \mathrm{p} 13)$ to be the only structural aberration among "pure" trisomies and tetrasomy 20 (Fig. 3). Monosomy 22, the most frequent chromosome aberration in meningiomas as a whole, was rare among hyperdiploid tumors. The usual cytogenetic markers of meningioma progression, i.e., further autosomal monosomies and monosomy $1 \mathrm{p}$, did not occur in this hyperdiploid series (Fig. 4).

Within the numerical aberrations among hyperdiploid meningiomas, a non-random although not uniform pattern emerged. While chromosomes 1, 2, 21, and the sex chromosomes were not involved, supernumerary copies of chromosomes $5,12,17$, and 20 were present in more than half of the cases, among these trisomy 12 observed most frequently with $13 / 16$ cases. In particular, combined trisomy 5-12-20 occurred in 8/16 cases, and $5-12-17-20$ in $6 / 16$ cases.

The two fibrous meningiomas were the only specimens with loss of chromosome 22 . Furthermore, they had gains of $(3,5,12$, and 17 in common.

Table 3 Comparison between cytogenetic subgroups regarding recurrence rate and clinical/histopathological features indicating an aggressive behavior

\begin{tabular}{lrrrr}
\hline Clinical feature & \multicolumn{4}{l}{ Cytogenetic subgroups broken down by GPS } \\
\cline { 2 - 5 } & GPS group 0 & GPS group 1 & GPS group 2 & Hyperdiploid group \\
\hline Recurrence & $27(7.9 \%)(n=343)$ & $11(4.0 \%)(n=273)$ & $15(33.3 \%)(n=45)$ & $2(12.5 \%)(n=16)$ \\
Tumor location at convexity & $83(24.2 \%)(n=343)$ & $92(33.7 \%)(n=273)$ & $23(51.1 \%)(n=45)$ & $10(62.5 \%)(n=16)$ \\
WHO grade II or III & $73(22.2 \%)(n=328)$ & $68(24.9 \%)(n=264)$ & $29(67.4 \%)(n=43)$ & $9(60.0 \%)(n=15)$ \\
\hline
\end{tabular}

GPS groups 0 and 1 refer to meningiomas without progression-associated chromosome changes, GPS group 2 refers to loss of $1 \mathrm{p}$ as the most significant established progression-associated cytogenetic aberration 
Fig. 2 (a) Karyogram of case no. 8; karyotype $54, \mathrm{XX},+3$, $+8,+9,+12,+13,+15,+20$, +20 . (b) Karyogram of case 15 ; karyotype $46, \mathrm{XY},+3,+5$, $+12,+17,+20,-22$

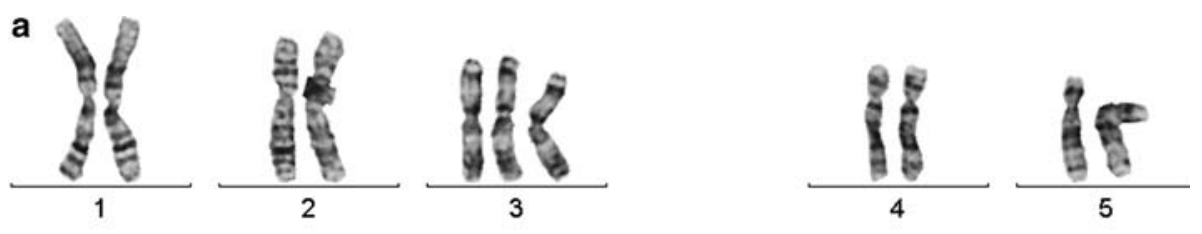

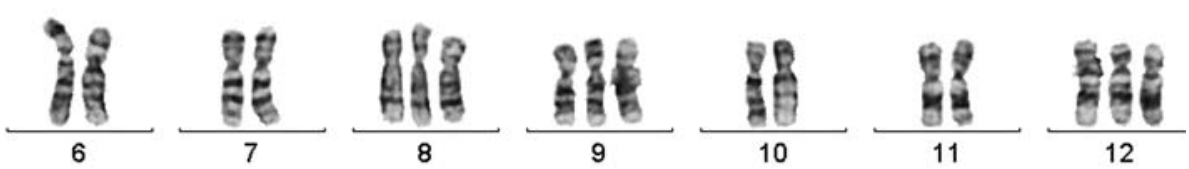
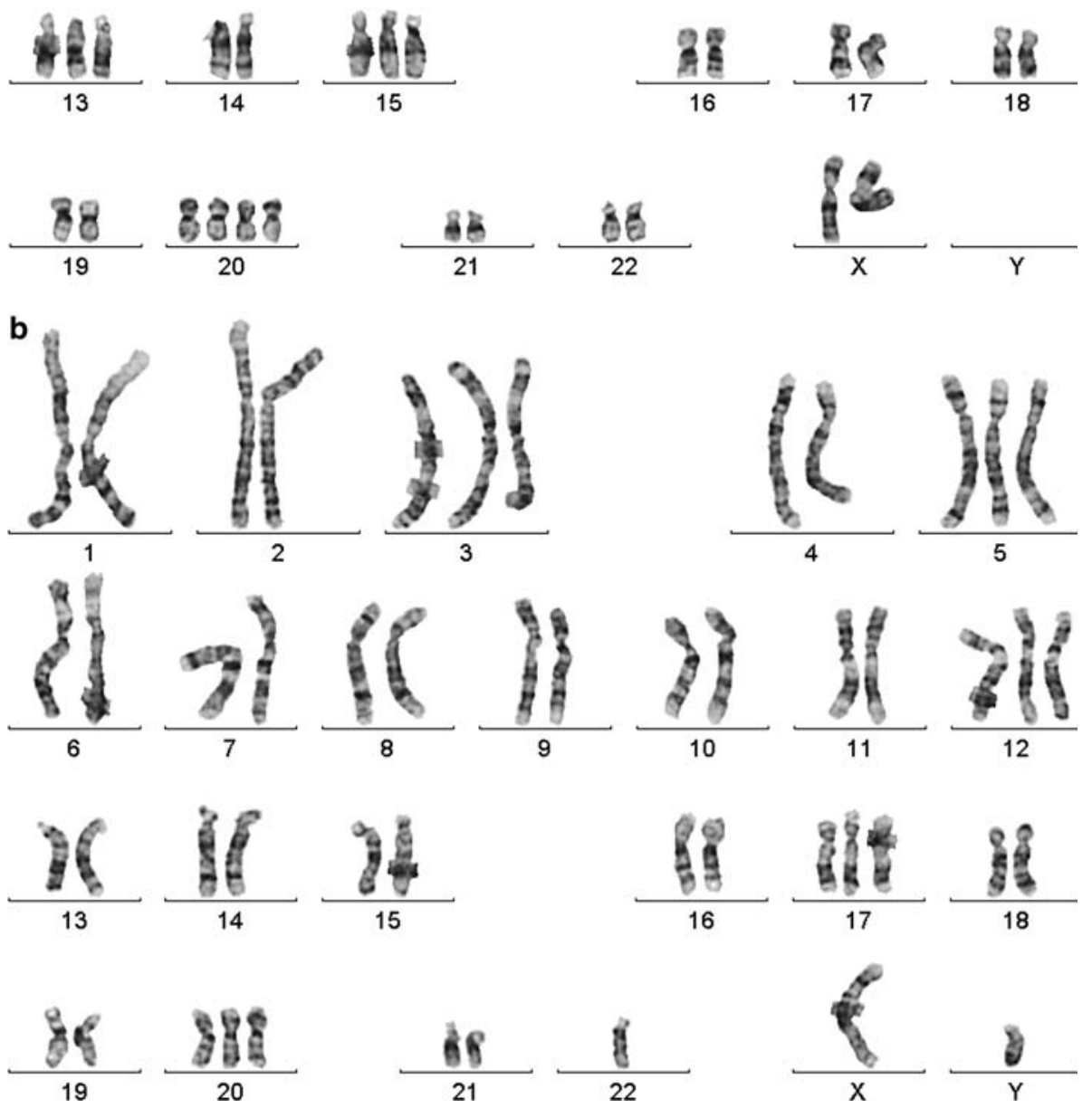

Fig. 3 Spectral karyotype of a hyperdiploid meningioma (case no. 16). Note the predominance of numerical aberrations; the only exception in this case is a $\operatorname{dic}(13 ; 16)(\mathrm{p} 13 ; \mathrm{p} 13)$ 


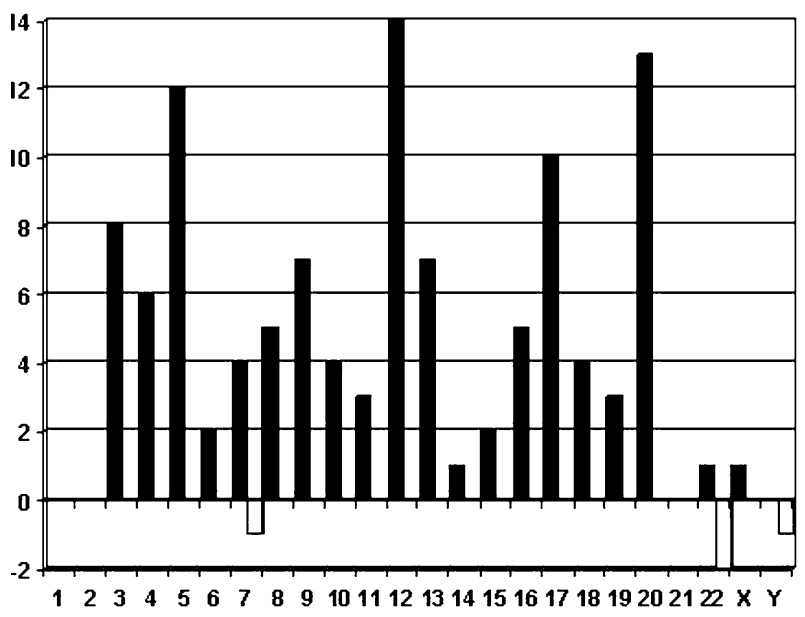

Fig. 4 Overview of numerical chromosome aberrations in 16 hyperdiploid meningiomas ( $X$-axis-no. of chromosome; $Y$-axis-no. of cases aberrant for the given chromosome). Note the presence of trisomy 5,12,17, and 20 each in more than half of the cases, with only two cases displaying monosomy 22

\section{Discussion}

Grading and prognostic assessment of meningiomas have always been controversial. The biological behavior of meningiomas can obviously not be accounted by histologic parameters alone. In 1979, Zülch stated that it is not the histological grading which is most crucial for the rate of recurrence of meningiomas, but primarily the completeness of extirpation [26]. There is consensus in the literature that radical surgical extirpation is strongly correlated with a favorable prognosis [9, 27].

Over 80 years ago, Cushing [28] reported on the frequency and behavior of meningiomas at different intracranial locations. Cytogenetic aberrations of meningiomas have later been shown to be significantly associated with the tumor location as well. Nearly half of the meningiomas of the cranial vault display progression-associated chromosome aberrations [4]. In 1980, Zankl and Zang showed that meningiomas of the skull base typically present with 46 chromosomes, whereas those of the convexity have significantly more chromosome aberrations [29]. It was already striking at that time that meningiomas which later recurred showed significantly more chromosome aberrations and a preferential localization at the convexity. These findings also apply to this present study, with $81 \%$ of the hyperdiploid tumors being located at the convexity, in contrast to $37 \%$ of cytogenetically typical meningiomas.

Cytogenetic patterns of meningiomas also may provide insight into their potential of recurrence. Numerical and structural chromosome changes with pronounced hypodiploidy, especially deletion of the short arm of a chromosome 1, are associated with more aggressive biological characteristics [30, 31].

In a previous study, we classified 198 consecutive meningiomas into four groups according to their karyotypes [4]. We found a very strong correlation between the cytogenetic findings and the rates of tumor recurrence.

Recurrences were found in $4.3 \%$ of tumors with normal diploid chromosome set or monosomy 22 as the sole cytogenetic aberration, $10.5 \%$ among markedly hypodiploid meningiomas, and the highest rate of recurrence was found in $30 \%$ of 40 tumors with deletions of the short arm of a chromosome 1 . These data fit well with the findings in our control group $(n=661)$, featuring a recurrence rate of $6.2 \%$ (normal or monosomy 22), and $33.3 \%$ belonging to the GPS group 2 . With recurrence in 2 of 16 hyperdiploid tumors presented here, this rare cytogenetic entity exhibits a higher recurrence rate than cytogenetically typical meningiomas but significantly less than meningiomas with deletion of one short arm of one chromosome 1 . Histomorphologically, all investigated hyperdiploid meningiomas lack typical meningothelial differentiation or specific morphological features. However, hyperdiploid meningiomas tend to histological dedifferentiation and progression. According to Perry et al. [20], brain invasion is not sufficient evidence for a malignant meningioma, but brain invasive histologically benign meningiomas behave similarly to atypical meningiomas in general, and it was stated as the strongest predictor of unfavorable outcome after surgically total resection. In Perry's series of 581 meningiomas, $4 \%$ were brain invasive. Compared to the hyperdiploid meningiomas described here, in 2/16 cases brain invasion was observed, thus the rate of invasion seems to be higher than in cytogenetically typical meningiomas. This feature, by itself, indicates a higher risk of recurrence and worse outcome for this cytogenetic meningioma subgroup.

Hyperdiploidy seems to be associated not only with loss of structural differentiation and with microcystic degeneration, but also with fibrous growth pattern: only those two cases with loss of chromosome 22 show a persistently fibrous and storiform growth pattern. Mutations in the neurofibromatosis gene 2 (NF2) on chromosome 22 occur significantly more frequently in fibrous and transitional meningiomas than in other subtypes, thus indicating a different molecular pathogenesis of the different phenotype variants of meningiomas [32].

Alkaline phosphatase (ALPL, liver/bone/kidney type) is coded as a single copy gene on chromosome 1p36.1-p34 and is a marker enzyme for the progression- 
associated loss of a putative tumor suppressor gene on chromosome 1p [21]. Thus, a focal or complete loss of alkaline phosphatase activity indicates tumor progression. Recently, Bouvier et al. [33] demonstrated that alteration of alkaline phosphatase is strongly associated with recurrence in meningiomas. Within this series of hyperdiploid meningiomas, in each tumor at least focal loss of enzyme activity, in three cases even complete loss was found. That is another point stressing the higher biological aggressiveness of the hyperdiploid meningiomas compared to the cytogenetically typical meningiomas. Nevertheless, in cytogenetic analysis no microscopically detectable disturbances of chromosome 1 could be recognized at all. We therefore suggest that a submicroscopic chromosomal defect or inactivation by methylation on (1p encompassing the region of the alkaline phosphatase gene may result in the loss of enzyme activity. Anyway, the reduced expression of alkaline phosphatase in hyperdiploid meningiomas supports the notion of increased growth potential of this entity.

Accordingly, we conclude that hyperdiploid meningiomas, with their non-random pattern of chromosomal trisomies and tetrasomies, constitute a cytogenetic entity of meningiomas with clinical and biological features of intermediate aggressiveness but without anaplasia. In the absence of microscopically detectable structural chromosome rearrangements, the increased growth potential of these meningiomas may be conferred by gene dosage imbalances rather than by fusion genes, deletions, or amplifications. Consequently, unlike in tumors with recurrent chromosomal breakpoints, no direct molecular cytogenetic strategy is at hand to determine the proliferation-enhancing gene or genes specifically involved in hyperdiploid meningiomas. Anyway, patients in whom a hyperdiploid meningioma has been recognized should be incorporated into an intensified regime of long-term postoperative monitoring.

Acknowledgments We would like to thank S. Urbschat, S. Wemmert, U. Bechtel, and U. Lindemann for expert technical assistance and helpful discussions. Financial support was provided by the BMBF (grant No.01GR0453 to JR) and HomFor (grant No. 68-06). The work at the Max-Planck-Institute for Informatics was performed in the context of the BioSapiens Network of Excellence (EU contact no. LSHG-CT-2003-503265).

\section{References}

1. Kujas M (1993) Meningioma. Curr Opin Neurol Neurosurg 6:882-887

2. Bello MJ, de Campos JM, Vaquero J, Kusak ME, Sarasa JL, Rey JM, Pestana A (1993) Chromosome 22 heterozygosity is retained in most hyperdiploid and pseudodiploid meningiomas. Cancer Genet Cytogenet 66:117-119
3. Bello MJ, de Campos JM, Kusak ME, Vaquero J, Sarasa JL, Pestana A, Rey JA (1994) Allelic loss at 1p is associated with tumor progression of meningiomas. Genes Chromosomes Cancer 9:296-298

4. Ketter R, Henn W, Niedermayer I, Steilen-Gimbel H, König J, Zang KD, Steudel WI (2001) Predictive value of progression-associated chromosome aberrations for the prognosis of meningiomas: a retrospective study of 198 cases. J Neurosurg 95:601-607

5. Kolles H, Niedermayer I, Schmitt C, Henn W, Feld R, Steudel WI, Zang KD, Feiden W (1995) Triple approach for diagnosis and grading of meningiomas: histology, morphometry of Ki-67/Feulgen stainings, and cytogenetics. Acta Neurochir 137:174-181

6. Zang KD (1982) Cytological and cytogenetical studies on human meningioma. Cancer Genet Cytogenet 6:249-274

7. Sayagues JM, Tabernero MD, Maillo A, Espinosa A, Rasillo A, Diaz P, Ciudad J, Lopez A, Merino M, Goncalves JM, Santos-Briz A, Morales F, Orfao A (2004) Intratumoral patterns of clonal evolution in meningiomas as defined by multicolor interphase fluorescence in situ hybridization (FISH): is there a relationship between histopathologically benign and atypical/anaplastic lesions?. J Mol Diagn 6:316-325

8. Zankl H, Singer H, Zang KD (1971) Cytological and cytogenetical studies on brain tumors. II. Hyperdiploidy, a rare event in human primary meningiomas. Humangenetik 11:253-257

9. Adegbite AB, Khan MI, Paine KWE, Tan LK (1983) The recurrence of intracranial meningiomas after surgical treatment. J Neurosurg 58:51-56

10. Arnoldus EP, Wolters LB, Voormolen JH, van Diunen SG, Raap AK, van der Ploeg M, Peters AC (1992) Interphase cytogenetics: a new tool for study of genetic changes in brain tumors. J Neurosurg 76:997-1003

11. Scholz M, Gottschalk J, Striepecke E, Firsching R, Harders A, Füzesi L (1996) Intratumorous heterogeneity of chromosomes 10 and 17 in meningiomas using non-radioactive in situ hybridization. J Neurosurg Sci 40:17-23

12. Maillo A, Diaz P, Sayagues JM, Blanco A, Tabernero MD, Ciudad J, Lopez A, Goncalves JM, Orfao A (2001) Gains of chromosome 22 by fluorescence in situ hybridization in the context of an hyperdiploid karyotype are associated with aggressive clinical features in meningioma patients. Cancer 92:377-385

13. Steudel WI, Feld R, Henn W, Zang KD (1996) Correlation between cytogenetic and clinical findings in 215 human meningiomas. Acta Neurochir Suppl 65:73-76

14. Olivecrona H (1934) Die parasagittalen Meningiome. Thieme, Leipzig

15. Simpson D (1957) The recurrence of intracranial meningiomas after surgical treatment. J Neurochem 20:22-39

16. Limon J, Dal Cin P, Sandberg AA (1986) Application of longterm collagenase disaggregation for the cytogenetic analysis of human solid tumors. Cancer Genet Cytogenet 23:305-313

17. Schröck E, Padilla-Nash H (2000) Spectral karyotyping and multicolor fluorescence in situ hybridization reveal new tumor-specific chromosomal aberrations. Semin Hematol 37:334-347

18. Louis DN, Scheithauer BW, Budka H, von Deimling A, Kepes JJ (2000) Meningiomas. In: Kleihues P, Cavenee WK (eds) Pathology and genetics of tumours of the nervous system. IARC, Lyon, pp 176-184

19. Perry A, Stafford SL, Scheithauer BW, Suman VJ, Lohse CM (1998) The prognostic significance of MIB-1, p53 and DNA flow cytometry in completely resected primary meningiomas. Cancer 82:2262-2269 
20. Perry A, Gutmann DH, Reifenberger G (2004) Molecular pathogenesis of meningiomas. J Neurooncol 70:183-302

21. Niedermayer I, Feiden W, Henn W, Steilen-Gimbel H, Steudel WI, Zang KD (1997) Loss of alkaline phosphatase activity in meningiomas: a rapid histochemical technique indicating progression-associated deletion of a putative tumor suppressor gene on the distal part of the short arm of chromosome 1. J Neuropathol Exp Neurol 56:879-886

22. Kim Y, Romeike B, Uszkoreit J, Feiden W (2005) The ImageJ based program and its description are downloadable at the morphometry section of http://www.uniklinikum-saarland.de/ neuropathologie

23. Beerenwinkel N, Rahnenführer J, Däumer M, Hoffmann D, Kaiser R, Selbig J, Lengauer T (2005) Learning multiple evolutionary pathways from cross-sectional data. J Comput Biol 12(6):584-598

24. Beerenwinkel N, Rahnenführer J, Kaiser R, Hoffmann D, Selbig J, Lengauer T (2005) Mtreemix: a software package for learning and use mixture models of mutagenetic trees. Bioinformatics 21(9):2106-2107

25. Rahnenführer R, Beerenwinkel N, Schulz WA, Hartmann C, von Deimling A, Wullich B, Lengauer T (2005) Estimating cancer survival and clinical outcome based on genetic tumor progression scores. Bioinformatics 21:2438-2446

26. Zülch KJ (1979) Histological typing of tumors of the central nervous system. World Health Organization, Geneva
27. Menon AG, Rutter JL, von Sattel JP, Snyder H, Murdoch C, Blumenfeld A, Martuza RL, von Deimling A, Gusella JF, Houseal TW (1997) Frequent loss of chromosome 14 in atypical and malignant meningioma: identification of a putative "tumor progression" locus. Oncogene 14:611-616

28. Cushing H (1922) The cranial hyperostoses produced by meningeal endotheliomas. Arch Neurol Psychiatry 8:139-152

29. Zankl H, Zang KD (1980) Correlation between clinical and cytogenetical data in 180 human meningiomas. Cancer Genet Cytogenet 1:351-356

30. Simon M, von Deimling A, Larson JJ, Wellenreuther R, Kaskel P, Waha A, Warnick RE, Tew JM Jr, Menon AG (1995) Allelic loss on chromosomes 14, 10 and 1 in atypical and malignant meningiomas: a genetic model of meningioma progression. Cancer Res 55:4696-4701

31. Zang KD (2001) Meningioma: a cytogenetic model of a complex benign human tumor, including data on 394 karyotyped cases. Cytogenet Cell Genet 293:207-220

32. Wellenreuther R, Kraus JA, Lenartz D, Menon G, Schramm J, Louis DN, Ramesh V, Gusella JF, Wiestler OD, von Deimling A (1995) Analysis of the neurofibromatosis 2 gene reveals molecular variants of meningioma. Am J Pathol 146:827-832

33. Bouvier C, Liprandi A, Colin C, Giorgi R, Quilichini B, Metellus P, Figarella-Branger D (2005) Lack of alkaline phosphatase activity predicts meningioma recurrence. Am J Clin Pathol 124:252-258 